\title{
EVOLUTION OF THE CORONAE IN EARLY-TYPE GALAXIES
}

\author{
L.P. David, W. Forman, and C. Jones \\ Harvard-Smithsonian Center for Astrophysics, Cambridge, MA, USA
}

\begin{abstract}
We present numerical simulations of the gaseous coronae in elliptical galaxies. These models consist of a modified King profile for the luminous portion of the galaxy and an isothermal dark halo. We include evolving stellar mass loss from planetary nebulae, and type I and II supernovae. Our models show that elliptical galaxies are likely to produce strong galactic winds at early times with $\mathrm{x}$-ray luminosities of $10^{42}-10^{44} \mathrm{ergs} \mathrm{s}^{-1}$ and temperatures of $10 \mathrm{keV}$. Galaxies can lose approximately $10-30 \%$ of their initial luminous mass in the wind which has an oxygen-to-iron ratio twice the solar value. Since elliptical galaxies are a principle component of rich clusters and compact groups this early wind phase affects the metallicity and temperature of the intracluster medium.
\end{abstract}

\section{INTRODUCTION}

Elliptical galaxies were thought to be nearly devoid of gas prior to the launch of the Einstein observatory. Constraints on the amount of gas in elliptical galaxies through optical and radio observations indicated that the gas mass was well below that which should accumulate through normal stellar evolutionary mass loss over the life time of a galaxy (Faber and Gallagher 1976). Due to the lack of cool gas in elliptical galaxies, most early theoretical studies were concerned with mechanisms for removing the stellar mass loss. Mathews and Baker (1971) showed that galactic winds may currently exist in elliptical galaxies which can efficiently remove all stellar mass loss. Observations with the Einstein satellite led to the discovery that $\mathrm{x}$-ray emission from hot $\left(T \approx 10^{7} \mathrm{~K}\right)$ extended coronae is nearly ubiquitous among earlytype galaxies (Forman, Jones, and Tucker 1985). The present x-ray luminosity of elliptical galaxies ranges from $10^{39}-10^{42} \mathrm{ergs} \mathrm{s}^{-1}$ in the $0.5-4.5 \mathrm{keV}$ band pass and is correlated with the optical luminosity (Forman, Jones, and Tucker 1985; Canizares, Fabbiano, and Trinchieri 1987). The observed x-ray luminosities are four orders of magnitude greater than the galactic wind models, indicating that the hot coronae must be in approximate hydrostatic equilibrium with the gravitational potential of the galaxy. The radiative cooling time of the gas within the hot coronae is typically less than a Hubble time. If heating is unable to balance cooling, this gas must cool as it settles into the center of the galaxy, and establish a cooling flow (Fabian and Nulsen 1977; Cowie and Binney 1977).

There have been a number of steady-state galaxy cooling flow models presented in the literature (White and Chevalier 1984; Sarazin and White 1987; Vedder, Trester, and Canizares 1988). These papers have extensively examined the effect of heavy halos, supernova rate, mass deposition, and environment on the present x-ray properties of elliptical galaxies. Recently, Loewenstein and Mathews (1987) 
have developed models of the evolution of the hot coronae in elliptical galaxies and find that accretion flows are common at late times. These models begin at a time corresponding to the main sequence life time of a $5 M_{\odot}$ star and hence do not include the consequences of type II supernovae at early epochs.

In this paper we present models for the evolution of the coronae in elliptical galaxies which begin shortly after the formation of the stellar component of the galaxy. Our models calculate the evolving rate of stellar mass loss from type I and II supernovae, and planetary nebulae. We find that the high rate of type II supernovae at early times generates hot, $\mathrm{x}$-ray luminous galactic winds that enrich the intracluster medium with heavy metals. We discuss the consequences of these early winds in clusters and the overall $x$-ray luminosity evolution of the coronae.

\section{METHOD}

In this section we briefly outline our method for simulating the evolution of the coronae in elliptical galaxies (see David, Forman, and Jones (1989), hereafter DFJ, for a more detailed development). We present the results of three different galaxy models with present optical luminosities of $10^{9}, 10^{10}$, and $10^{11} L_{\odot}$. Each galaxy model consists of a modified King profile for the luminous portion of the galaxy along with an isothermal dark halo which comprises $90 \%$ of the total mass of the galaxy. The stellar population is assumed to form instantaneously without any residual gas remaining. The stars are assumed to form with a Salpeter initial mass function with an upper mass cutoff of $40 M_{\odot}$. Stellar mass loss occurs instantaneously as stars evolve off the main sequence. All stars more massive than $8 M_{\odot}$ develop into type II supernovae and leave behind $1.4 M_{\odot}$ remnants. Stars less massive than this lose mass through a planetary nebula and leave behind a remnant with a mass given by the expression in Tinsley (1976). The evolving rate of type I supernovae is determined using the method discussed in Matteucci and Greggio (1986) and is normalized to the rate given in van den Bergh et al. (1987) at the present epoch. All of this information is then incorporated into a time-dependent, spherically symmetric, hydrodynamics code in order to determine the evolving dynamical state of the interstellar medium in elliptical galaxies.

\section{X-RAY LUMINOSITY EVOLUTION}

The evolution of the corona in an elliptical galaxy is completely governed by the evolving stellar mass loss rates, and the mass-averaged temperature of the injected gas. At early times, gas is injected into a galaxy with a temperature of $\sim 10^{8} \mathrm{~K}$ due to the high rate of type II supernovae (see DJF). Once all stars more massive than $8 M_{\odot}$ have evolved off the main sequence, the temperature of the injected gas drops precipitously. At late times the temperature of the injected gas increases gradually since the mass injection rate from planetary nebulae decreases more rapidly than the mass injection rate from type I supernovae. We find that the interstellar medium evolves through three dynamically distinct stages in these models. These are: 1) 
an initial galactic wind phase generated by the high rate of type II supernovae, 2) a transition phase during which the interstellar medium evolves from a pure wind to a pure accretion flow as the rate of type II supernovae diminishes, and $3 \mathrm{a}$ ) a cooling flow phase which endures for a Hubble time in optically luminous galaxies, or $3 \mathrm{~b}$ ) a second wind phase which commences at late times as the mass-averaged temperature of the injected gas surpasses the escape temperature in low luminosity models $\left(\leqslant 10^{9} L_{\odot}\right)$.

The evolution of the $\mathrm{x}$-ray luminosity of a corona is primarily determined by the central gas density since the $\mathrm{x}$-ray emissivity is proportional to $\rho^{2} T^{1 / 2}$ and the gas temperature only varies by a factor of 10 over a Hubble time. Figure 1 shows that the x-ray luminosity increases at first as gas is injected into the galaxy, and attains a maximum value when the mass injection rate is balanced by the mass loss rate in the wind. The $\mathrm{x}$-ray luminosity during the wind phase is $100-1000$ times greater than the present epoch $x$-ray luminosity of elliptical galaxies and the wind temperature is $10 \mathrm{keV}$. Extending the formation of the stellar population over $10^{8}$ years reduces the peak $x$-ray luminosity during the wind phase by approximately an order of magnitude (see DJF). As the wind continues to evacuate the galaxy, the central density and $x$-ray luminosity decrease, reaching a minimum value at the start of the transition phase. As the gas begins accreting into the center of the galaxy, the $\mathrm{x}$-ray luminosity increases reaching a second maximum at the start of the cooling flow phase. At late times $L_{x}$ decreases due to the continually decreasing stellar mass loss rate. The sharp drop in the x-ray luminosity in the $10^{9} L_{\odot}$ model (see figure) is due to the development of a galactic wind at late times. In models which maintain a cooling flow, the $x$-ray luminosity at late times can be approximated by $L_{x} \propto t^{-0.9}$.

\section{CONSEQUENCES OF THE EARLY WIND PHASE}

The observation of X-ray emission lines from cluster gas requires that a significant amount of elements synthesized in stellar interiors are injected into the intracluster medium. The enrichment of the intracluster medium can result from both galactic winds and ram pressure stripping. Assuming that all galaxies within a cluster form with a Salpeter IMF and develop winds which remove all of the metal enriched type II supernovae ejecta, we can estimate the metallicity of the cluster gas from the results given in DFJ. All galaxies lose approximately $10-30 \%$ of their initial luminous mass during the wind phase. The mass fraction yield of Fe produced by type II supernovae in a galaxy with a Salpeter IMF is $5 \times 10^{-4}$ (DFJ). Using these values, the iron abundance of the cluster gas relative to the solar value will be $0.30 M_{\text {stars }} / M_{\text {gas }}$ after the cessation of the early wind phase, where $M_{\text {stars }}$ is the initial stellar mass of the cluster and $M_{\text {gas }}$ is the initial gas mass in the cluster (the residual gas remaining after galaxy formation ends). Significant amounts of oxygen are produced in type II supernovae which result in an oxygen-to-iron abundance ratio after the wind phase which is approximately twice the solar value. Using a 


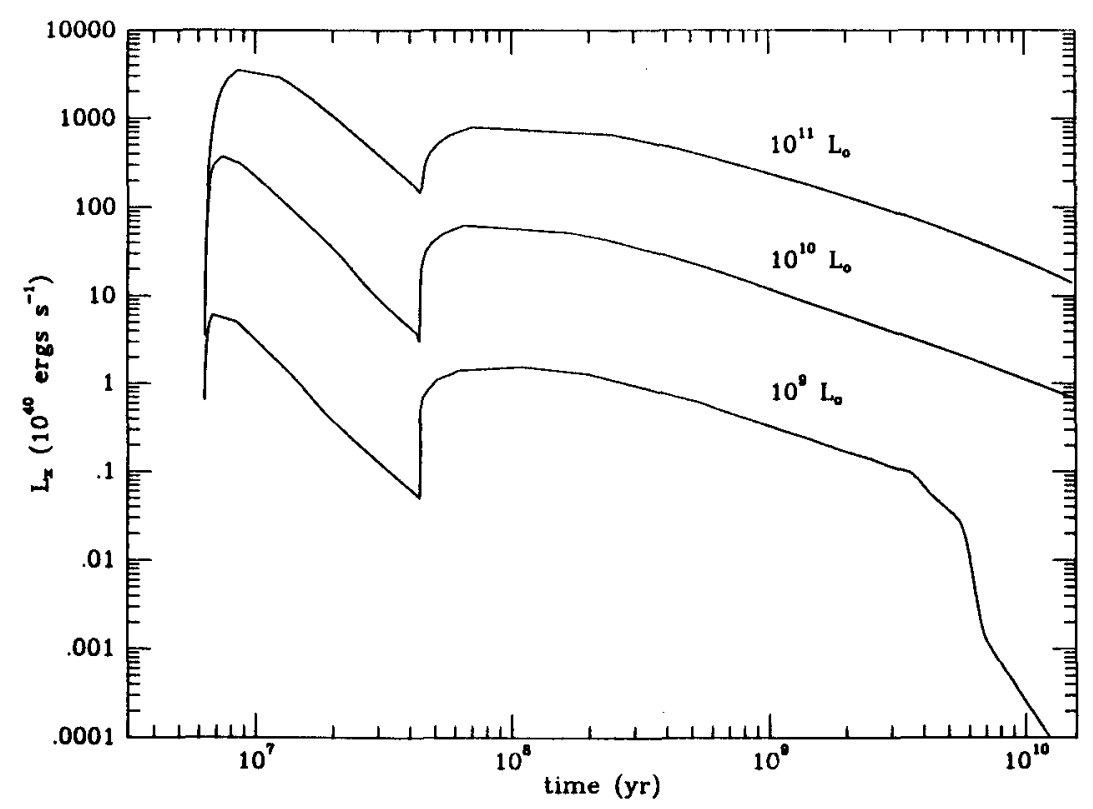

Fig 1. X-ray luminosity evolution of three galaxy models with different optical luminosities at the present epoch.

present supernovae rate equal to the rate given in van den Bergh et al. (1987), the mass fraction yield of $\mathrm{Fe}$ from type I supernovae is $6 \times 10^{-4}$. Assuming that ram pressure stripping is one hundred percent efficient in the removal of stellar mass loss, the resulting $\mathrm{Fe}$ abundance of the cluster gas after a Hubble time will be $0.66 M_{\text {star }} / M_{\text {gas }}$. Very little oxygen is produced in type I supernovae relative to type II so that ram pressuring stripping reduces the oxygen-to-iron abundance ratio to approximately the solar value. By determining the oxygen and iron abundances for clusters at different epochs with observations from future $x$-ray telescopes, the IMF of the first generation of stars and the efficiency of ram pressure stripping can be determined. Also, due to the observed decrease of $M_{\text {stars }} / M_{\text {gas }}$ with cluster richness (David et al. 1989; Jones et al., this proceedings), our models predict that the metallicity of the intracluster gas should decrease from poor to rich clusters.

A second important effect of galactic winds is to inject energy into the intracluster medium. The density distribution of the hot gas in many clusters of galaxies is well fit using isothermal hydrostatic $\beta$ models, where $\beta$ is the ratio of the energy per unit mass in galaxies to the specific internal energy of the gas. The best fitting values of $\beta$ range from $1 / 2$ for groups and poor clusters to $2 / 3$ for rich clusters. If the temperature of the gas and galaxies resulted from gravitational collapse in a cluster, these temperatures should be more nearly equal. One explanation for this discrepancy is heating of the cluster gas during the early galactic wind phase. From our models presented in DFJ, the mass-averaged temperature of the gas ejected in 
the wind is $1.6 \times 10^{8} \mathrm{~K}$. If the initial temperature of the cluster gas is assumed equal to the galaxy temperature, the net heating of the cluster gas can be determined. Using $\beta=T_{g a l} / T_{g a s}$, where $T_{g a l}$ is the galaxy temperature, and $T_{g a s}$ is the initial gas temperature, the fractional change in the temperature of the intracluster gas can be written as

$$
\beta=1-0.45\left(\frac{M_{\text {stars }}}{M_{\text {gas }}}\right)
$$

This expression indicates that ample heating of the cluster gas can be provided by early galactic winds. Since $M_{s t a r s} / M_{\text {gas }}$ decreases systematically from poor to rich clusters (Jones et al., this proceedings), our models predict that heating by galactic winds should produce a direct correlation between $\beta$ and cluster richness (see Forman et al. this proceedings for further discussion).

\section{CONCLUSIONS}

We find that the gas within elliptical galaxies evolves through three dynamically distinct stages. Our models indicate that a substantial portion of the heavy elements observed in the intracluster medium can be supplied during the early wind phase, and that the energy injected can alter the ratio of energy per unit mass in galaxies to that in gas. The high $\mathrm{x}$-ray luminosity and temperature during the early wind phase indicate that $\mathrm{x}$-rays are a prime region of the spectrum to search for primeval galaxies. If galaxies form at redshifts of 4 to 5 , the observed emission will be redshifted to $2 \mathrm{keV}$, an energy at which future $\mathrm{x}$-ray observatories will be very sensitive.

\section{REFERENCES}

Canizares, C.R., Fabbiano, G., and Trinchieri, G. 1987, Ap.J., 312, 503.

Cowie, L.L., and Binney, J. 1977, Ap.J., 215, 723.

David, L.P., Arnaud, K.A., Forman, W., and Jones, C. 1989 (preprint).

David, L.P., Forman, W., and Jones, C. 1989 (preprint).

Faber, S., and Gallagher, J. 1976, Ap.J., 204, 365.

Fabian, A.C., and Nulsen, P.E.J. 1977, MNRAS, 180, 479.

Forman, W., Jones, C., and Tucker, W. 1985, Ap.J., 293, 102.

Loewenstein, M., and Mathews, W. G. 1987, Ap.J., 319, 614.

Mathews, W.G., and Baker, J. 1971, Ap.J., 170, 241.

Matteucci, F., and Greggio, L. 1986, A.A., 154, 279.

Sarazin, C.S., and White ,R.E. 1987, Ap.J., 320, 32.

White, R.E., and Chevalier, R.A. 1984, Ap.J., 280, 561.

Tinsley, B.M. 1976, Ap.J., 208, 797.

van den Bergh, S., McClure, R., and Evans, R. 1987, Ap.J., 323, 44.

Vedder, P.W., Trester, J.J., and Canizares, C.R. 1988, Ap.J., 332, 725. 